\title{
Performance monitoring at the task and the response level
}

\author{
Charlotte Desmet $^{1,2, *}$, Wim Fias ${ }^{1,2}$ and Marcel Brass ${ }^{1,2}$ \\ ${ }^{1}$ Department of Experimental Psychology, Ghent \\ University, B-9000 Ghent, Belgium \\ ${ }^{2}$ Ghent Institute for Functional and Metabolic Imaging of \\ the brain, Ghent University, B-9000 Ghent, Belgium \\ *Corresponding author. \\ e-mail: charlotte.desmet@Ugent.be
}

\begin{abstract}
How errors and conflict are processed in the human brain, has been extensively investigated over the last decades. In this review, we argue that error research has mainly focused on one type of errors, namely errors at the response level. Furthermore, research on conflict and errors has primarily used a very restricted set of experimental paradigms, raising the question as to whether the results from this research can be generalized to other forms of errors and conflict. We thus argue to approach errors and conflict from a broader perspective.
\end{abstract}

Keywords: conflict; error; SRC task; task and response level.

\section{Introduction}

In daily life, we constantly adjust our behavior according to the flexible demands of the environment. Situations of conflict and errors form key aspects for these behavioral adjustments. Moreover, a situation of conflict, or an error, signals that the current behavior is no longer appropriate and that adjustments are needed. Over the past decades, an innumerable amount of studies on error and conflict monitoring have emerged. In the first part of this review, we will give a short overview of the classical research conducted on error and conflict processing. Because this literature has been extensively reviewed elsewhere (e.g., Botvinick et al., 2001; Ridderinkhof et al., 2004; Ullsperger and von Cramon, 2004), this overview will be very brief. Thereafter, we will show that current research has approached errors and conflict from a narrow point of view. More precisely, the classical literature has focused on one type of errors and on one type of experimental design.

\section{Response errors and response conflict}

In the laboratory, a range of stimulus-response compatibility (SRC) tasks, such as the flanker task (Eriksen and Eriksen, 1974) or the Simon task (Simon, 1969), have been frequently used to study conflict and errors. Typically, in these sorts of tasks, a conflict at the response level occurs. In other words, participants have to perform a response while irrelevant information is interfering with this correct response. For example, in the flanker task, the direction of a central arrow has to be determined (left or right). In a situation of conflict, the surrounding arrows will point in the opposite direction and consequently activate the wrong response. Due to this situation of response conflict, response errors will arise more frequently than in nonconflicting situations. Usually, response errors and response conflict are thus studied within the same experiments.

At a behavioral level, several robust post error and post conflict effects have been documented. First of all, it has been observed that participants are slower after an error than after a correct response. Post error slowing has served as evidence for an adjustment in control after an error (Laming, 1968; Botvinick et al., 2001). That is, after an error, participants will increase control to avoid errors in the future and therefore be slower on subsequent trials. Recently, however, it has been suggested that post error slowing can be seen as an attentional effect rather than as a control effect (Notebaert et al., 2009; Núñez Castellar et al., 2010). Secondly, a well established finding regarding post conflict processing, is the conflict adaptation effect. This refers to reduced interference effects after conflict trials compared to after no conflict trials. This effect, first described by Gratton and colleagues in the flanker task, has been interpreted as a cognitive control effect (Gratton et al., 1992). Namely, a conflict trial will ask for more control, resulting in a smaller interference effect on the next trial. The conflict adaptation effect has been replicated in a wide range of tasks, including Simon tasks (Sturmer et al., 2002), Stroop Tasks (Kerns et al., 2004), and prime-target congruency effects (Kunde, 2003). However, until now, it is still unclear to what extend stimulus repetitions can account for the adaptation effect (Mayr et al., 2003; Mayr and Awh, 2009; Funes et al., 2010).

At a neural level, a negative deflection, occurring $50 \mathrm{~ms}$ after the onset of a response error, has been well documented. This event-related potential (ERP) component is assumed to reflect conflict between concurring responses (Botvinick et al., 2001) or to signal an outcome that is worse than expected (Holroyd and Coles, 2002) and has been labeled the error related negativity (ERN). Further, the rostral cingulate zone (RCZ) has been suggested to be the main generator of the ERN (Ullsperger and von Cramon, 2001; Ridderinkhof et al., 2004). The neural correlates of response errors and response conflict have been reported in more detail in an enormous number of functional magnetic resonance imaging (fMRI) studies (Ridderinkhof et al., 2004). The posterior frontomedian cortex (pFMC), and in particular the RCZ is considered as being extremely crucial for adaptive control (Ullsperger and von 
Cramon, 2001, 2004; Ridderinkhof et al., 2004). Whether, errors and conflict are processed in the same brain regions, however, is still an open issue. Some studies have shown that the RCZ is activated both by errors and conflict (Carter et al., 1998; Kerns et al., 2004). This is in accordance with the conflict monitoring theory (Botvinick et al., 2001). This theory states that the RCZ will be activated whenever two responses compete. That is, both in the cases of response conflict and response errors. In this view, response errors are thus special cases of response conflict. However, others have reported a distinction in the pFMC between errors and conflict. These latter studies showed more ventral parts related to response errors and more dorsal parts of the pFMC related to response conflict (Kiehl et al., 2000; Braver et al., 2001; Ullsperger and von Cramon, 2001; Wittfoth et al., 2008). This relation is in accordance with the connections of the pFMC to motor output. In particular, ventral parts of the $\mathrm{pFMC}$ are more related to the primary motor cortex and the spinal cord, whereas dorsal parts are connected to brain areas related to high-level motor cognition (Ullsperger and von Cramon, 2001, 2004). Since errors trigger the tendency to perform the correct response, it seems plausible that they are more related to regions correlated with motor output than response conflict.

\section{Beyond the response level: task conflict and task errors}

To this point, we have described literature concerning errors and conflict at a response level. However, conflict does not only occur at the response level, but can also occur at the task level. Surprisingly, the literature on task conflict is completely separated from the literature on response conflict. Two large domains of task conflict studies can be distinguished. First of all, several authors have used the Stroop paradigm (Stroop, 1935; Macleod, 1991) to study processes related to task conflict. In the Stroop task, participants are required to name the color in which a word is printed while ignoring the meaning of the word (which is also a color). In a conflict situation, the meaning of the word and the color in which it is printed, will not correspond. In other words, a conflict at a task level emerges (word naming versus color naming). Brain regions associated with these situations of conflict, are the anterior cingulate cortex (ACC) and the presupplementary motor area (preSMA) (Milham and Banich 2005; Woodward et al., 2008; Aarts et al., 2009). It should be noted, though, that in these paradigms, an influence of response related conflict cannot be ruled out entirely because the two tasks (color naming and word naming) share the same responses (e.g., green and red). This issue was addressed by van Veen and Carter (2005). They also used a Stroop task, but mapped two colors on one response (for example the colors red and yellow should be answered by pressing the left button). In this way they could disentangle task conflict from response conflict. More precisely, the color naming task and the word naming task could indicate different outcomes while at a response level no conflict emerged (the same response button is required for both tasks). These authors found a more dorsal part of the pFMC activated by task conflict (defined as semantic conflict in their study) than by response conflict. Recent attempts showed that, also by means of an ex-Gaussian distribution analysis, both types of conflict could be separated in the Stroop task (Steinhauser and Hübner, 2009).

A different class of paradigms investigating task conflict are the task switching paradigms (Monsell, 2003). In these kinds of paradigms, people perform two tasks randomly or in a predetermined order. A robust finding in task switching experiments is the switch costs. That is, longer response times and more errors occur on task switch trials relative to task repetition trials (e.g., Monsell, 2003). In task switching studies, adaptive behavior at the task level thus concentrates on the control processes needed to alternate between different tasks. Many studies have found a fronto-parietal network to be involved in task switching. This network includes the left inferior frontal junction, the left posterior superior parietal lobule (Ruge et al., 2005), regions along the inferior frontal sulcus, the left intraparietal sulcus, the anterior insula (Dove et al., 2000), the ACC (Hyafil et al., 2009) and the preSMA (Brass and von Cramon, 2002; Rushworth et al., 2002; Crone et al., 2006). Importantly, task switching does not only require resolution of conflict at the task level, but also involves other processes such as goal setting and response related processes (e.g., Rubinstein et al., 2001). Therefore, the task switching paradigm does not provide a pure measure of task conflict.

In summary, research on adaptation at the response level has concentrated on response conflict and response errors, typically measured in SRC tasks. On the other hand, conflict at the task level has been studied with Stroop and task switching paradigms. Surprisingly, to our knowledge no brain imaging study has ever investigated the neural correlates of task errors. So far, only behavioral studies have addressed this issue. Steinhauser and Hübner (2006) compared switch effects after response errors with switch effects after task errors. They observed normal switch costs after response errors. However, after task errors, switch benefits instead of switch costs emerged. The authors explained this finding by arguing that at the moment of a task error, the wrong task is strongly activated. Consequently, subsequent task switches will actually represent task repetitions and therefore switch benefits instead of switch costs appear after a task error. In further experiments, Steinhauser and Hübner (2006) confirmed that the moment of response execution is crucial for task strengthening to occur. More specifically, they demonstrated switch costs after corrected task errors, but switch benefits after detected but uncorrected task errors. In a recent experiment, we replicated the dissociation between task errors and response errors on switch cost. However, in addition, we showed that a task error occurring before response execution also influences subsequent switch costs. In other words, preparing or selecting the wrong task without executing it, will also influence future task performance. In summary, at a behavioral level, there is evidence that response and task errors have a different impact on subsequent behavior (Desmet, Fias, and Brass, unpublished data).

As mentioned above, task errors have never been investigated at the neural level. Given the extensive debate 
regarding the similarity/dissimilarity between response errors and response conflict, this is very surprising. From a neuroanatomical perspective, it is not clear whether one would expect a dissociation of task and response errors. On the one hand, there are overlapping brain areas for response and task processing (i.e., the RCZ). On the other hand, there are also brain areas uniquely related to task processing. To gain further insight into the relation of errors and conflict at response and task level, we recently investigated response errors, response conflict, task errors and task conflict in one experiment (Desmet et al., 2011). Our data thus allow us to dissociate errors and conflict at the task and the response level. In our experiment, participants had to perform a letter and a color task in a random order. A cue indicated which task had to be performed (the word 'color' or 'letter' appeared prior to target onset). During the letter task, the target letter had to be classified as L or R. During the color task the color of the target letter had to be classified as green or yellow. Three trial types were presented. In catch trials, only a target letter was presented after cue presentation. In task change trials, a vertical ellipse appeared around the target letter. This ellipse indicated that participants did not have to perform the task as indicated by the cue, but had to apply the other task. For example, if a cue indicated to do the letter task and subsequently an ellipse appeared around the target, the color task had to be applied. A task error was defined as the application of the wrong task to the target letter. The time between target presentation and the appearance of the ellipse was adjusted according to a staircase algorithm. In this way, the amount of task errors was around $50 \%$. Finally, in stimulus change trials, we offered a second stimulus after target presentation. This second stimulus always elicited a different response than the target stimulus. However, the same task had to be applied. For example, after presentation of the letter cue, the target stimulus ' $L$ ' could change into a second stimulus 'R'. Again, the time between the second stimulus and the target stimulus was adjusted to a staircase procedure so that the amount of response errors was also set at $50 \%$. For an overview of the different trial types see Figure 1.

We used univalent stimulus-response mappings. In particular, every possible outcome ('L', 'R', 'green' or 'yellow') was mapped onto a different finger. Further, the two different tasks were divided over both hands. In this way, we could infer from the subjects' response which error was made. For example, the response 'green' to a green R under execution of the letter task would comprise a task error. We found a dissociation of both error types in the pFMC: while response errors activate the RCZ, task errors activate a more dorsal part of the pFMC, labeled the dorsal frontomedian cortex. Further, a clear dissociation was perceived between brain areas related to conflict at the task and response level. In particular, as regards activity in the pFMC, task conflict was related to activation in the

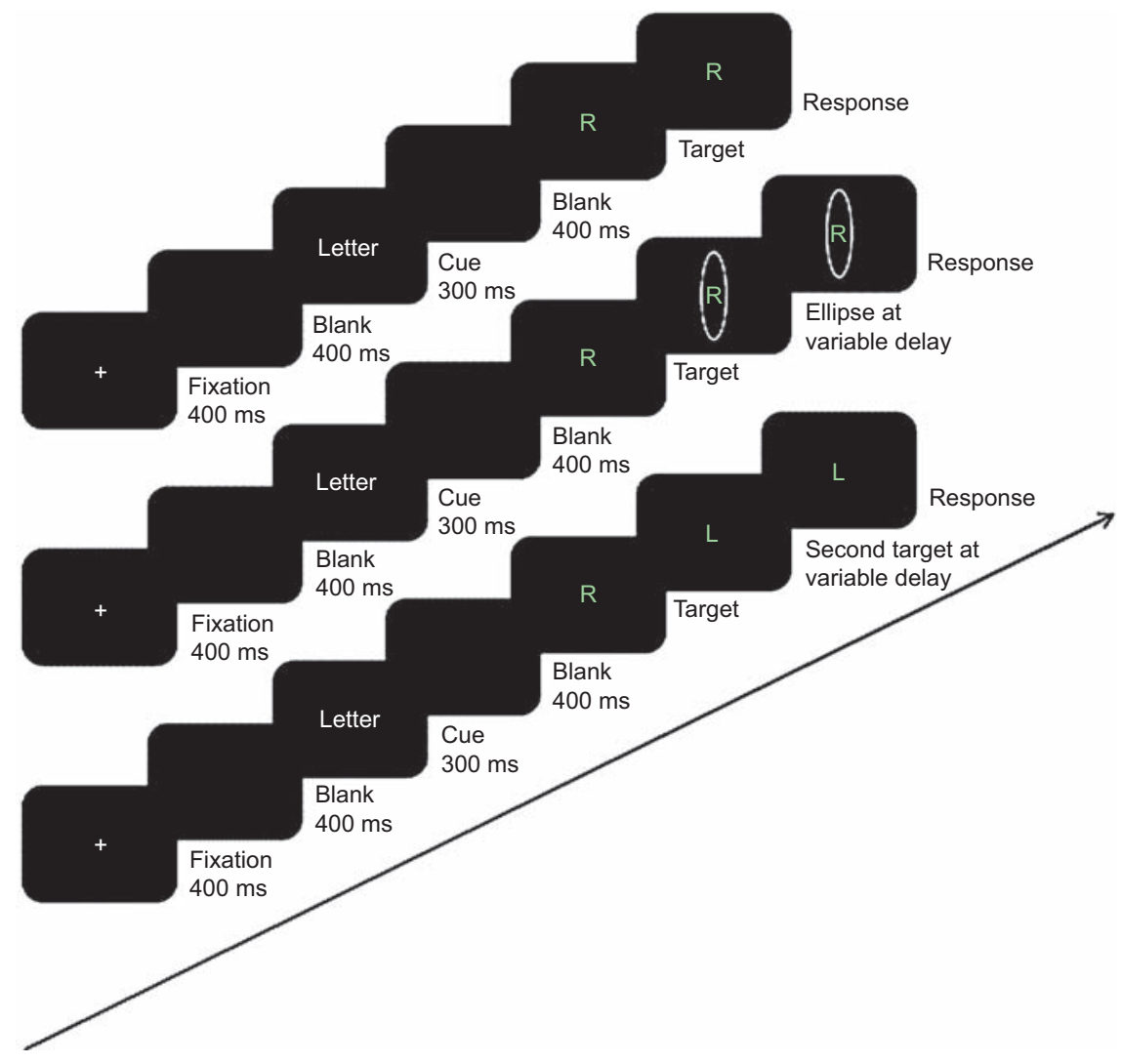

Figure 1 Overview of the three trial types.

From top to bottom: catch trial, task change trial and stimulus change trial. 
preSMA, while response conflict was related to activation in the RCZ. In general, this study demonstrated that task related processing requires more dorsal parts of the pFMC than response related processing. Further, error related processing is related to more anterior parts of the pFMC than conflict related processing. Finally, within each level of abstractness, conflict was always more posterior and more dorsally located than errors (Figure 2) (Desmet et al., 2011).

There are some papers that address different sub localizations in the pFMC. However these studies are restricted to conflict research. For example, some studies have shown distinctions between forms of pre-response conflict and response conflict. It seems that pre-response conflict is associated with more dorsal and posterior parts of the pFMC than response conflict (Kim et al., 2011). Others have shown an anterior to posterior typology, ranging from strategy control, over decision control, towards response control (Venkatraman et al., 2009). Also, decision conflict without response conflict seems to activate regions in the pFMC more dorsal than those usually found for response conflict (Pochon et al., 2008). More related to our distinction of task and response conflict, Orr and Weissman (2009) presented a cue and a target in two modalities at the same time (visual and auditory modality). At cue level, the visual modality informed participants which feature of the target (look or hear) they should react to. The target comprised a spoken and a visually presented ' $\mathrm{x}$ ' or ' $\mathrm{o}$ '. The authors found that conflict between the visual and the auditory information at a cue level (which represents a conflict between tasks) activated dorsal parts of the pFMC while conflict at a target level (which represents conflict at the response level) activated ventral parts of the pFMC.

So, while different levels of conflict (see also Egner, 2008) and their corresponding brain areas have been discussed in

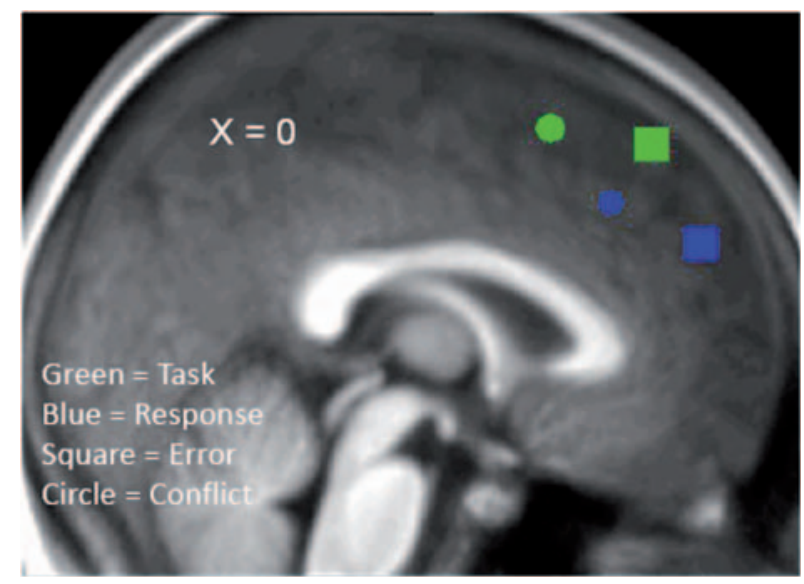

Figure 2 Peak activations in the pFMC for task errors (MNI coordinates of maximal random-effect $Z$ scores, $x, y, z=6,39,54$, $Z=4.45$ ), task conflict (MNI coordinates of maximal random-effect $Z$ scores, $x, y, z=-9,15,57, Z=4.03$ ), response errors (MNI coordinates of maximal random-effect $Z$ scores, $x, y, z=6,51,30, Z=3.87$ ) and response conflict (MNI coordinates of maximal random-effect $\mathrm{Z}$ scores, $x, y, z=-6,30,39, Z=3.70$ ). the literature, the story for different levels of error processing is less extensive. Previously, Krigolson and Holroyd (2007) reported a dissociation between high and low level errors. They manipulated these two levels of error processing, in a movement tracking task. Participants were required to move a cursor from a start point to a target point by manipulating a joystick. However, the target point sometimes changed to a different location, resulting in low level errors. In some cases, participants could not move the cursor, making it impossible to achieve the movement goal. These cases were labeled as high level errors. The authors showed by means of ERP measurements, that the frontal brain system is related to high level errors, while the posterior system is correlated with low level errors. Apart from this study and our own experiment, there seems to be no research concerning the sub localization of errors in the brain. However, the task error region that we described in the pFMC, has been described before in higher level decision processes (Elliott and Dolan, 1998; Goel and Dolan, 2000; Rushworth et al., 2002; Volz et al., 2003, 2004). Based on these findings, and keeping in mind the different connections of the pFMC to motor output, we argued that more abstract errors are processed in more dorsal regions of the pFMC.

\section{Errors and conflict from a broader perspective}

So far, we have given an overview of studies on conflict and error processing at the task and the response level. However, almost all of these studies investigate very simple tasks that have limited ecological validity. As outlined above, conflict and/or error processing, has been studied in different spatial and non-spatial compatibility tasks such as the flanker task, the Stroop task and the Simon task. Even though a wide range of paradigms have been used, adaptive behavior in these simplified tasks is restricted to very specific strategies. In the flanker task, for example, an error occurs when the central arrow is categorized incorrectly. The only possible strategy to prevent this error in subsequent trials, is to pay more attention to the central arrow. However, in many real life situations, an error will not only cause an increase in attention, but will also provide information that can be used to optimize further behavior. When mastering a sport such as dancing or playing a musical instrument, it is crucial to learn from errors, in the sense that one needs to analyze what is going wrong. This learning aspect of errors is somewhat neglected in typical SRC tasks. As a consequence, current accounts of error monitoring might underestimate the cognitive operations following error detection. Therefore, it is crucial to investigate adaptive behavior in tasks that require more complex adaptive strategies in the case of an error. In a recent experiment, we addressed this issue by examining if the same post error adaptation effects described in SRC tasks are also present when performing a mental arithmetic task. Participants had to judge whether the solution of a multiplication problem was correct or not (for example $4 \times 6=24$ ). Conflict was manipulated by the relatedness between the solution and the problem. For example, ' $4 \times 6=21$ ' is a low conflict trial, since 21 
is not related to the multiplication table of 4 , nor to the table of 6 . In contrast, ' $4 \times 6=28$ ' is a high conflict trial, since 28 is related to the table of $4(4 \times 7=28)$. We observed post error accuracy increases and post error slowing. Although from a control perspective, post accuracy increases are expected (more control is required after an error resulting in a slower and better performance), they are not commonly observed in SRC tasks (Rabbitt and Rodgers, 1977; Laming, 1979; Hajcak et al., 2003; Hajcak and Simons, 2008). Consequently, we argued that tasks, where reflection about an error is possible, will induce more than an orientation response and will therefore, besides post error slowing, also cause post error accuracy increases. Further, we did not find evidence for conflict adaptation. We reasoned that conflict adaptation might also be an effect typically related to SRC tasks. More specifically, a recent model of conflict adaptation states that only stimulus-response rules active at the moment of conflict will be strengthened (Verguts and Notebaert, 2009). Since the stimulus set in mental arithmetic is substantially larger than the stimulus set in a SRC task, strengthening a particular arithmetic problem will not be beneficial on subsequent trials. For example, strengthening ' $4 \times 6=24$ ' will not be beneficial if on the next trial ' $3 \times 5=15$ ' is presented (Desmet, De Brauwer, Imbo, Brass, Fias and Notebaert, unpublished). Given these behavioral differences of adaptive processes in SRC tasks and tasks that require more complex adaptive processes, it might be interesting to investigate if there is also a neural distinction between these more 'cognitive' arithmetic errors and errors resulting from classical SRC tasks.

\section{Conclusion}

Adaptive control has been widely studied in cognitive neuroscience over the last two decades. This research, however, is dominated by investigating a specific type of error and conflict in a very restricted set of experimental paradigms. Here, we argue that it is necessary to investigate adaptive control in a wider range of experimental tasks and to distinguish adaptive control at different levels.

Firstly, we showed that brain regions associated with errors and conflict at more abstract levels of processing can be distinguished from brain regions related to response related processing. One important question for future research is how behavior is adapted when conflict and errors occur at a more abstract level such as the task level. Both behavioral data (Steinhauser and Hübner, 2008) as well as brain imaging data (Desmet et al., 2011) suggest that it is important to distinguish adaptive control at the task and the response level.

Secondly, we provided evidence that behavioral effects that have been extensively described and that have served as evidence for human adaptive control, might be restricted to a specific class of experimental paradigms. When using a mental arithmetic task instead of a SRC task, the behavioral adaptation effects changed profoundly.

To conclude, considering the wide variety in which errors occur in daily life, the focus of current studies of error and conflict monitoring is relatively narrow. As described above, highlighting different aspects of error and conflict processing, changes the neural and behavioral results. Thus, in order to make correct extrapolations about the human ability to process errors and conflict and to gain further insight into these processes, a broader research perspective should be used.

\section{Acknowledgments}

This work was supported by a grant from FWO (G.0630.08) and by the Belgian Science Policy, Interuniversity Attraction Poles program (P6/29). We acknowledge the support of Ghent University (Multidisciplinary Research Partnership 'The integrative neuroscience of behavioral control').

\section{References}

Aarts, E., Roelofs, A., and van Turennout, M. (2009). Attentional control of task and response in lateral and medial frontal cortex: brain activity and reaction time distributions. Neuropsychologia 47, 2089-2099.

Botvinick, M.M., Braver, T.S., Barch, D.M., Carter, C.S., and Cohen, J.D. (2001). Conflict monitoring and cognitive control. Psychol. Rev. 108, 624-652.

Brass, M. and von Cramon, D.Y. (2002). The role of the frontal cortex in task preparation. Cereb. Cortex 12, 908-914.

Braver, T.S., Barch, D.M., Gray, J.R., Molfese, D.L., and Snyder, A. (2001). Anterior cingulate cortex and response conflict: effects of frequency, inhibition and errors. Cereb. Cortex 11, 825-836.

Carter, C.S., Braver, T.S., Barch, D.M., Botvinick, M.M., Noll, D., and Cohen, J.D. (1998). Anterior cingulate cortex, error detection, and the online monitoring of performance. Science 280, 747-749.

Crone, E.A., Wendelken, C., Donohue, S.E., and Bunge, S.A. (2006). Neural evidence for dissociable components of task-switching. Cereb. Cortex 16, 475-486.

Desmet, C., Fias, W., Hartstra, E., and Brass, M. (2011). Errors and conflict at the task level and the response level. J. Neurosci. 31, 1366-1374.

Dove, A., Pollmann, S., Schubert, T., Wiggins, C.J., and von Cramon, D.Y. (2000). Prefrontal cortex activation in task switching: an event-related fMRI study. Cognit. Brain Res. 9, 103-109.

Egner, T. (2008). Multiple conflict-driven control mechanisms in the human brain. Trends Cognit. Sci. 12, 374-380.

Elliott, R. and Dolan, R.J. (1998). Activation of different anterior cingulate foci in association with hypothesis testing and response selection. Neuroimage 8, 17-29.

Eriksen, B.A. and Eriksen, C.W. (1974). Effects of noise letters upon identification of a target letter in a nonsearch task. Percept. Psychophys. 16, 143-149.

Funes, M.J., Lupianez, J., and Humphreys, G. (2010). Analyzing the generality of conflict adaptation effects. J. Expert Psychol. Hum. Percept. Perform. 36, 147-161.

Goel, V. and Dolan, R.J. (2000). Anatomical segregation of component processes in an inductive inference task. J. Cogn. Neurosci. $12,110-119$.

Gratton, G., Coles, M.G H., and Donchin, E. (1992). Optimizing the use of information-strategic control of activation of responses. J. Exp. Psychol. Gen. 121, 480-506. 
Hajcak, G., McDonald, N., and Simons, R.F. (2003). To err is autonomic: error-related brain potentials, ANS activity, and post-error compensatory behavior. Psychophysiology 40, 895-903.

Hajcak, G. and Simons, R.F. (2008). Oops!.. I did it again: an ERP and behavioral study of double-errors. Brain Cognit. 68, 15-21.

Holroyd, C.B. and Coles, M.G.H. (2002). The neural basis of human error processing: reinforcement learning, dopamine, and the error-related negativity. Psychol. Rev. 109, 679-709.

Hyafil, A., Summerfield, C., and Koechlin, E. (2009). Two Mechanisms for task switching in the prefrontal cortex. J. Neurosci. 29, 5135-5142.

Kerns, J.G., Cohen, J.D., MacDonald, A.W., Cho, R.Y., Stenger, V.A., and Carter, C.S. (2004). Anterior cingulate conflict monitoring and adjustments in control. Science 303, 1023-1026.

Kiehl, K.A., Liddle, P.F., and Hopfinger, J.B. (2000). Error processing and the rostral anterior cingulate: an event-related fMRI study. Psychophysiology 37, 216-223.

Kim, C., Kroger, J.K., and Kim, J. (2011). A functional dissociation of conflict processing within anterior cingulate cortex. Hum. Brain Mapp. 32, 304-312.

Krigolson, O.E. and Holroyd, C.B. (2007). Hierarchical error processing: different errors, different systems. Brain Res. 1155, 70-80.

Kunde, W. (2003). Sequential modulations of stimulus-response correspondence effects depend on awareness of response conflict. Psychon. Bull. Rev. 10, 198-205.

Laming, D.R. (1968). Information theory of choice-reaction times. London: Academic Press.

Laming, D.R. (1979). Choice reaction performance following an error. Acta Psychol. 43, 199-224.

Macleod, C.M. (1991). Half a century of research on the stroop effect - An integrative review. Psychological Bulletin 109, 163-203.

Mayr, U., Awh, E., and Laurey, P. (2003). Conflict adaptation effects in the absence of executive control. Nat. Neurosci. 6, 450-452.

Mayr, U. and Awh, E. (2009). The elusive link between conflict and conflict adaptation. Psychol. Res.-Psychologische Forschung 73, 794-802.

Milham, M.P. and Banich, M.T. (2005). Anterior cingulate cortex: an fMRI analysis of conflict specificity and functional differentiation. Hum. Brain Mapp. 25, 328-335.

Monsell, S. (2003). Task switching. Trends in Cogn. Sci. 7, 134-140.

Notebaert, W., Houtman, F., Van Opstal, F., Gevers, W., Fias, W., and Verguts, T. (2009). Post-error slowing: an orienting account. Cognition 111, 275-279.

Nunez Castellar, E., Kuhn, S., Fias, W., and Notebaert, W. (2010). Outcome expectancy and not accuracy determines posterror slowing: ERP support. Cogn. Affect. Behav. Neurosci. 10, 270-278.

Orr, J.M. and Weissman, D.H. (2009). Anterior Cingulate cortex makes 2 contributions to minimizing distraction. Cereb. Cortex 19, 703-711.

Pochon, J.B., Riis, J., Sanfey, A.G., Nystrom, L.E., and Cohen, J.D. (2008). Functional imaging of decision conflict. J. Neurosci. 28, 3468-3473.

Rabbitt, P.M.A. and Rogers, R.D. (1977). What does a man do after he makes an error? An analysis of response programming. Q. J. Exp. Psychol. 29, 727-743.

Ridderinkhof, K.R., Ullsperger, M., Crone, E.A., and Nieuwenhuiss, S. (2004). The role of the medial frontal cortex in cognitive control. Science 306, 443-447.

Ridderinkhof, K.R., van den Wildenberg, W.P.M., Segalowitz, S.J., and Carter, C.S. (2004). Neurocognitive mechanisms of cognitive control: the role of prefrontal cortex in action selection, response inhibition, performance monitoring, and reward-based learning. Brain Cognit. 56, 129-140.

Rubinstein, J.S., Meyer, D.E., and Evans, J.E. (2001). Executive control of cognitive processes in task switching. J. Exp. Psychol. Hum. Percept. Perform. 27, 763-797.

Ruge, H., Brass, M., Koch, I., Rubin, O., Meiran, N., and von Cramon, D.Y. (2005). Advance preparation and stimulus-induced interference in cued task switching: further insights from BOLD fMRI. Neuropsychologia 43, 340-355.

Rushworth, M.F.S., Hadland, K.A., Paus, T., and Sipila, P.K. (2002). Role of the human medial frontal cortex in task switching: a combined fMRI and TMS study. J. Neurophysiol. 87, 2577-2592.

Simon, J.R. (1969). Reactions toward source of stimulation. J. Exp. Psychol. 81, 174-176.

Steinhauser, M. and Hübner, R. (2006). Response-based strengthening in task shifting: evidence from shift effects produced by errors. J. Exp. Psychol. Hum. Percept. Perform. 32, 517-534.

Steinhauser, M. and Hübner, R. (2008). How task errors affect subsequent behavior: evidence from distributional analyses of taskswitching effects. Memory Cognit. 36, 979-990.

Steinhauser, M. and Hübner, R. (2009). Distinguishing response conflict and task conflict in the stroop task: evidence from exgaussian distribution analysis. J. Exp. Psychol. Hum. Percept. Perform. 35, 1398-1412.

Stroop, J.R. (1935). Studies of interference in serial verbal reactions. J. Exp. Psychol. 18, 643-662.

Sturmer, B., Leuthold, H., Soetens, E., Schroter, H., and Sommer, W. (2002). Control over location-based response activation in the simon task: behavioral and electrophysiological evidence. J. Exp. Psychol. Hum. Percept. Perform. 28, 1345-1363.

Ullsperger, M. and von Cramon, D.Y. (2001). Subprocesses of performance monitoring: a dissociation of error processing and response competition revealed by event-related fMRI and ERPs. Neuroimage 14, 1387-1401.

Ullsperger, M. and von Cramon, D.Y. (2004). Neuroimaging of performance monitoring: error detection and beyond. Cortex 40 , 593-604.

Van Veen, V. and Carter, C.S. (2005). Separating semantic conflict and response conflict in the Stroop task: a functional MRI study. Neuroimage 27, 497-504.

Venkatraman, V., Rosati, A.G., Taren, A.A., and Huettel, S.A. (2009). Resolving response, decision, and strategic control: evidence for a functional topography in dorsomedial prefrontal cortex. J. Neurosci. 29, 13158-13164.

Verguts, T. and Notebaert, W. (2009). Adaptation by binding: a learning account of cognitive control. Trends Cognit. Sci. 13, 252-257.

Volz, K.G., Schubotz, R.I., and von Cramon, D.Y. (2003). Predicting events of varying probability: uncertainty investigated by fMRI. Neuroimage 19, 271-280.

Volz, K.G., Schubotz, R.I., and von Cramon, D.Y. (2004). Why am I unsure? Internal and external attributions of uncertainty dissociated by fMRI. Neuroimage $21,848-857$.

Wittfoth, M., Kustermann, E., Fahle, M., and Herrmann, M. (2008). The influence of response conflict on error processing: evidence from event-related fMRI. Brain Res. 1194, 118-129.

Woodward, T.S., Metzak, P.D., Meier, B., and Holroyd, C.B. (2008). Anterior cingulate cortex signals the requirement to break inertia when switching tasks: a study of the bivalency effect. Neuroimage 40, 1311-1318.

Received May 26, 2011; accepted July 5, 2011 


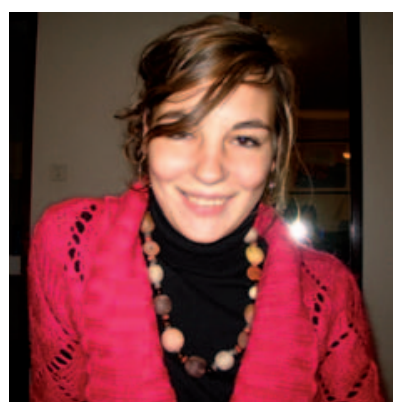

Charlotte Desmet received her master's degree at Ghent University in 2007. Since then she has worked at the Department of Experimental Psychology at Ghent University. In 2008 she started a $\mathrm{PhD}$ under the supervision of Marcel Brass and Wim Fias. She is examining the role of errors at different levels of abstractness using behavioral and fMRI experiments.

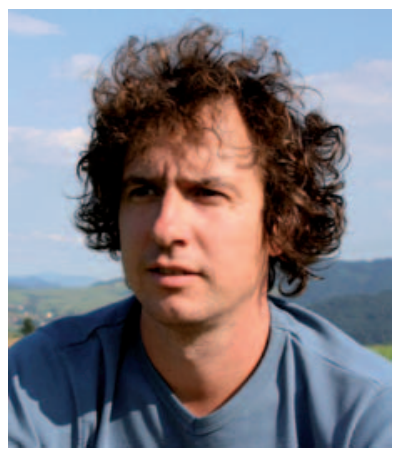

Wim Fias obtained his $\mathrm{PhD}$ in Experimental Psychology at KULeuven. He then moved to Ghent University where he joined the Department of Experimental Psychology. He directs the Ghent Institute for Neuroscience. His main interests are numerical cognition and cognitive control which he studies with behavioral experiments, fMRI, EEG and TMS.

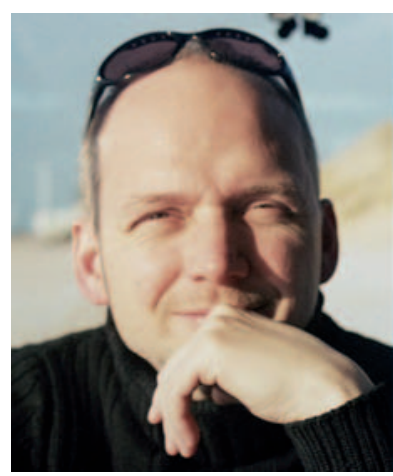

Marcel Brass did his $\mathrm{PhD}$ at the Max Planck Institute for Psychological Research in Munich (Germany). Then he worked as a research scientist at the Max Planck Institute for Human Cognitive and Brain Sciences in Leipzig (Germany). Since 2006 he holds a research professorship at the Department of Experimental Psychology at Ghent University (Belgium). Marcel Brass investigates cognitive control, intentional action and the link of perception and action from a neuro-cognitive perspective. 